\title{
Erratum to: 47. Jahrestagung der Österreichischen Gesellschaft für Innere Medizin
}

\section{Kathrin Hoffmann}

Published online: 22 November 2016

(C) Springer-Verlag Wien 2016

\section{Erratum to:}

Wien Klin Wochenschr

DOI 10.1007/s00508-016-1086-6.

For abstract P26 "Meet the needs: Important questions for ICU relatives" the co-authors and affiliations are missing.

The complete authorship with their affiliations are as follows:

\author{
M. M. Hoffmann ${ }^{1,6}$ \\ A. K. Holl ${ }^{2}$ \\ H. Burgsteiner ${ }^{3}$ \\ T. R. Pieber ${ }^{1,4}$ \\ P. Eller ${ }^{5}$ \\ K. Amrein ${ }^{1}$
}

${ }^{1}$ Medizinische Universität Graz, Universitätsklinik für Innere Medizin - Klinische Abteilung für Endokrinologie und Diabetologie, Graz, Austria

${ }^{2}$ Medizinische Universität Graz, Universitätsklinik für Psychiatrie, Graz, Austria

${ }^{3}$ FH Joanneum, Gesundheitsinformatik / E-Health, Graz, Austria

${ }^{4}$ Joanneum Research, Institut HEALTH, Graz, Austria ${ }^{5}$ Universitätsklinik für Innere Medizin - Internistische Intensivstation, Graz, Austria

${ }^{6}$ LKH- Univ. Klinikum Graz, Qualitätsmanagement und Risikomanagement, Graz, Austria

The online version of the original article can be found under http://dx.doi.org/10.1007/s00508-016-1086-6. 